\title{
Impact of global hemodynamic load on exercise capacity in aortic stenosis 2 , 败弥
}

\author{
R. Dulgheru ${ }^{\text {a,b }}$, J. Magne ${ }^{\text {a }}$, R. Capoulade ${ }^{\text {c }}$, L. Davin ${ }^{\text {a }}$, D. Vinereanu ${ }^{\text {b }}$, L.A. Pierard ${ }^{\text {a }}$, \\ P. Pibarot ${ }^{\mathrm{C}}$, P. Lancellotti ${ }^{\mathrm{a}, *}$ \\ a University of Liège Hospital, GIGA Cardiovascular Sciences, Heart Valve Clinic, Department of Cardiology, CHU Sart Tilman, Liège, Belgium \\ ${ }^{\mathrm{b}}$ University of Medicine and Pharmacy 'Carol Davila', Bucharest, Romania \\ c Laval University, Department of Medicine, Quebec, Canada
}

\section{A R T I C L E I N F O}

\section{Article history:}

Received 27 June 2012

Received in revised form 23 October 2012

Accepted 18 January 2013

Available online 27 February 2013

\section{Keywords:}

Valve

Aortic stenosis

Valvulo-arterial impedance

Cardiopulmonary test

Functional capacity

\begin{abstract}
A B S T R A C T
Background: The determinants of maximal exercise capacity (MEC) in aortic stenosis (AS) are, in large part, unknown. We hypothesized that the left ventricular (LV) global hemodynamic load - as assessed by the valvulo-arterial impedance (Zva) - is one of the main determinants of MEC and we sought to evaluate the factors associated with reduced MEC in AS.

Method and results: Asymptomatic patients with moderate or severe AS ( $\mathrm{n}=62$, aortic valve area $<1.5 \mathrm{~cm}^{2}$, $65 \pm 13$ years, $68 \%$ men) and preserved LV systolic function (ejection fraction $>50 \%$ ) were prospectively referred for comprehensive resting echocardiography and cardiopulmonary exercise test. Absolute peak $\mathrm{VO}_{2}$ was $19.5 \pm 5.7 \mathrm{~mL} / \mathrm{kg} / \mathrm{min}$ (median $19.6 \mathrm{~mL} / \mathrm{kg} / \mathrm{min}$; range $7.2-33.1 \mathrm{~mL} / \mathrm{kg} / \mathrm{min}$ ). There were significant correlations between peak $\mathrm{VO}_{2}$ and: age, body mass index, LV stroke volumes, cardiac output, mean flow rate, mitral annulus $\mathrm{s}^{\prime}$ and $\mathrm{e}^{\prime}$ wave velocities, $\mathrm{E} / \mathrm{e}^{\prime}$ ratio and left atrial diameter (all $\left.\mathrm{p}<0.05\right)$. Indexed mean flow rate and Zva were the strongest univariable echocardiographic determinants of peak $\mathrm{VO}_{2}(\mathrm{r}=0.44, \mathrm{p}<0.001$ and $\mathrm{r}=-0.39, \mathrm{p}=0.002$, respectively). In addition, patients with reduced $\mathrm{MEC}$ ( peak $\mathrm{VO}_{2}<$ median) had higher Zva than those with preserved MEC ( $4.24 \pm 1.18$ vs. $\left.3.71 \pm 0.68 \mathrm{~mm} \mathrm{Hg} / \mathrm{mL} / \mathrm{m}^{2}, \mathrm{p}=0.036\right)$. In multivariable analysis, age $(\mathrm{p}<0.001)$ and $\mathrm{Zva}(\mathrm{p}=0.048)$ were the only independent predictors $\left(\mathrm{r}^{2}=0.40\right)$ of peak $\mathrm{VO}_{2}$. Conclusion: In asymptomatic patients with moderate to severe AS, MEC varies widely among patients, and is often lower than expected. Global LV hemodynamic load is the main echocardiographic determinant of reduced MEC in these patients, suggesting its usefulness for their clinical evaluation and management.
\end{abstract}

(C) 2013 Elsevier Ireland Ltd. All rights reserved.

\footnotetext{
is Contributorship statement: RD: study design, echocardiographic examination recording, echocardiographic exams analysis, clinical data recording, data analysis and drafting of the manuscript. This author takes responsibility for all aspects of the reliability and freedom from bias of the data presented and their discussed interpretation; JM: study design, echocardiographic exams analysis, clinical data recording, data analysis and review of the manuscript; RC: echocardiographic exams analysis, clinical data recording and review of the manuscript; LD: echocardiographic exams analysis and review of the manuscript; DV: review of the manuscript; LAP: study design, echocardiographic examination recording, echocardiographic exams analysis, clinical data recording, data analysis and review of the manuscript; PP: study design, data analysis and review of the manuscript; PL: study design, echocardiographic examination recording, echocardiographic exams analysis, clinical data recording, data analysis and review of the manuscript.

is is $^{2}$ Source of funding: Dr. Dulgheru received a 1-year research scholarship from the Romanian Society of Cardiology. Dr. Magne is research associate from the F.R.S-FNRS, Brussels, Belgium and received grants from the Fonds Léon Fredericq, Liège, Belgium and from the Fond pour la Chirurgie Cardiaque, Belgium. Dr. Pibarot holds the Canada Research Chair in Valvular Heart Disease, Canadian Institutes of Health Research (CIHR), Ottawa, Ontario, Canada. This study was funded in part by research grant from CIHR (MOP\# 114997).

* Corresponding author at: Department of Cardiovascular Imaging, University Hospital Sart Tilman, B-4000 Liege, Belgium. Tel.: + 324366 71 94; fax: + 3243667195 .

E-mail address: plancellotti@chu.ulg.ac.be (P. Lancellotti).
}

\section{Introduction}

Valvular aortic stenosis (AS) is the most common cardiovascular disease in developed countries after coronary artery disease and hypertension. AS cannot be viewed as an isolated disease of the valve [1]. Indeed, arterial compliance is often reduced due to atherosclerosis and medial elastocalcinosis in AS patients [2]. Reduced arterial compliance adds to the hemodynamic load caused by AS and contributes to the global burden imposed on the LV. Hence, the LV of AS patients often faces a double hemodynamic load: valvular + arterial. Briand et al. have proposed a new Doppler-echocardiographic parameter: the valvulo-arterial impedance (Zva) to assess the global load imposed on the LV in such patients. This parameter incorporates the valvular load and the pulsatile and steady components of the arterial load [3]. High Zva has been associated with increased risk of LV dysfunction [3] and cardiovascular events in both symptomatic and asymptomatic AS patients. The impairment of maximal exercise capacity (MEC) should logically be related to the global hemodynamic burden faced by the LV. We thus hypothesized that Zva is an important factor limiting MEC in AS patients.

Although, exercise testing is largely recommended in asymptomatic AS, its ability to differentiate pathological from physiological 
breathlessness is limited, especially in the elderly subjects [4]. Moreover, even if the test is absolutely normal - 'truly' asymptomatic patients - there is still a high inter-patient variability in terms of outcome [5]. Altogether, this has led to different levels of recommendations for aortic valve replacement in the ACC/AHA and ESC guidelines for the management of asymptomatic AS (class IIb vs I for exerciseinduced symptoms). Cardiopulmonary exercise testing (CPET) with determination of gas exchange provides quantitative, objective, and more accurate non-invasive evaluation of MEC [6]. Various factors may affect MEC in patients with AS. However, very few studies have focused on evaluating MEC and its determinants in AS. In the present study, we sought to evaluate the determinants of MEC in asymptomatic AS patients and to test the hypothesis that a high Zva plays an important detrimental role on MEC in such patients.

\section{Methods}

\subsection{Study population}

Sixty-five consecutive patients with asymptomatic moderate or severe AS who were able to perform a treadmill CPET were prospectively included in the study. Patients were recruited from two centres, University Hospital Sart Tilman Liège $(n=33)$ and Québec Heart \& Lung Institute $(n=32)$. Exclusion criteria: (1) more than mild aortic or mitral regurgitation at echocardiography, (2) abnormal cardiac rhythm, (3) documented second degree or third degree atrio-ventricular block or paced rhythm, (4) dilated or hypertrophic obstructive cardiomyopathy, (5) known ischemic heart disease, (6) left ventricular (LV) ejection fraction $<50 \%$, (7) primary pulmonary hypertension, (8) evidence of more than mild pulmonary disease and (9) inadequate acoustic windows. For the CPET, the absolute exclusion criteria specified by ATS/ACCP Statement on Cardiopulmonary Testing were also followed [6]. All patients gave their consent. The study protocol conforms to the ethical guidelines of the 1975 Declaration of Helsinki as reflected in a priori approval by the institution's human research committee.

\subsection{Echocardiographic measurements}

Echocardiographic examination was performed using a Vivid ultrasound (7 or E9) System (GE Healthcare, Little Chalfont, UK). All data were stored in a digital format, which enabled ulterior off-line analysis using dedicated software (EchoPAC, GE Vingmed Ultrasound AS, Horten, Norway).

\subsubsection{Evaluation of AS severity}

Continuous wave Doppler was used to measure trans-aortic velocities. Peak and mean trans-aortic pressure gradients were calculated using the simplified Bernoulli equation $\left(\Delta \mathrm{P}=4 \mathrm{v}^{2}\right.$, where $\mathrm{v}$ is maximal aortic velocity in $\mathrm{m} / \mathrm{s}$ ). Aortic valve area was calculated using the continuity equation. AS was considered severe if the valve area was $<1 \mathrm{~cm}^{2}$

\subsubsection{Evaluation of $L V$ geometry, $L V$ systolic and diastolic function}

The LV end-diastolic and end-systolic diameters were measured from the 2D parasternal long-axis view. The LV mass was calculated using the formula of the American Society of Echocardiography and was indexed for body surface area. The LV ejection fraction, end-systolic and end-diastolic volumes were measured by Simpson's biplane method. The LV stroke volume was calculated by multiplying the LV outflow tract area to LV outflow tract velocity time integral measured by pulse wave Doppler. Mean flow rate across the aortic valve was calculated by dividing stroke volume by ejection time from the same cardiac cycle. Stroke volume and mean flow rate were indexed to body surface area. The LV cardiac output was calculated as the product of heart rate and stroke volume and was indexed to body surface area to derive the cardiac index. Peak E-wave and A-wave velocities at the level of the LV inflow were measured using pulsed-wave Doppler. Peak systolic $\left(\mathrm{s}^{\prime}\right)$ and peak early diastolic $\left(\mathrm{e}^{\prime}\right)$ mitral lateral annular velocities were measured using tissue Doppler imaging in apical 4-chamber view. The E/ $\mathrm{e}^{\prime}$ ratio was then calculated. Left atrial antero-posterior diameter was measured from the 2D parasternal long-axis view.

\subsubsection{Assessment of global LV load}

The global LV hemodynamic load was estimated by the valvulo-arterial impedance using the following formula: $\mathrm{Zva}=(\mathrm{SBP}+\mathrm{MG}) / \mathrm{SVi}$, where $\mathrm{SBP}$ is the systolic blood pressure, measured at the time of the echocardiographic examination, MG the mean transvalvular pressure gradient and SVi the LV outflow tract stroke volume indexed for body size area. Zva represents the valvular and arterial factors that oppose ventricular ejection by absorbing the mechanical energy developed by the LV.

\subsection{Cardiopulmonary exercise testing}

All patients underwent a multistage symptom-limited treadmill CPET. A modified Bruce protocol was used, with three distinct stages: a warm up stage, an exercise stage with a progressive increase in workload each $3 \mathrm{~min}$ and a recovery stage. Warm-up stage started at a speed of $2.7 \mathrm{~km} / \mathrm{h}$ and an inclination of 5\%. During exercise stage, the speed of the treadmill increased with $1.3 \mathrm{~km} / \mathrm{h}$ for each stage and the inclination increased with $5 \%$ for the first stage, and with $2 \%$ for each remaining stage of the protocol. Each stage of the protocol lasted $3 \mathrm{~min}$. Patients were encouraged to exercise to exhaustion. Blood pressure values were carefully monitored at the end of each stage of the protocol using a calibrated sphygmomanometer, and continuous 12 lead ECG monitoring was also recorded. Patient's medications were not stopped for the test. A cardiologist closely monitored the patient during the test and carefully searched for symptom development or signs of abnormal test: angina, dizziness, sustained ventricular arrhythmias, drop or inadequate rise $(<20 \mathrm{~mm} \mathrm{Hg})$ in systolic blood pressure or $\geq 2 \mathrm{~mm}$ ST segment depression $80 \mathrm{~ms}$ after the J point. The test was promptly stopped in case of symptom development. Peak oxygen consumption (peak $\mathrm{VO}_{2}$ ) was measured with the use of a gas exchange analyzer (VMax Series 29c, SensorMedics). Peak heart rate (peak HR), peak SBP, peak diastolic blood pressure (peak DBP), oxygen pulse $\left(\mathrm{O}_{2}\right.$ pulse), peak circulatory power (PCP) and the metabolic equivalents (METs) were also measured. Peak circulatory power was calculated as the product of peak oxygen uptake (peak $\mathrm{VO}_{2}$ ) and peak $\mathrm{SBP}$, as previously validated. Device calibration was performed before each test. Each CPET was performed within 1 month following echocardiographic examination. Three patients were excluded from the study because of low peak $\mathrm{VO}_{2}$ values related to extraneous causes (rapid rise in blood pressure in one patient and lower limb pain in the other two).

The authors of this manuscript have certified that they comply with the principles of Ethical Publishing in the International Journal of Cardiology.

\subsection{Statistical analysis}

Patients were divided in two groups according to the median value of the peak $\mathrm{VO}_{2}$ distribution. The results are expressed as mean \pm standard deviation or percentages unless otherwise specified. Statistical differences between groups were assessed using Student $t$ test, chi-square test or Fisher exact test, as appropriate. Relationship between echocardiographic data and peak $\mathrm{VO}_{2}$ were evaluated using linear regression. To identify the independent predictors of exercise capacity, we used multiple linear regression analysis. Only variables univariately associated with peak $\mathrm{VO}_{2}$ were included into the model and were carefully chosen in order to avoid colinearity. Values of $p<0.05$ were considered significant. All statistical analyses were performed with SPSS version 17.0 (SPSS Inc., Chicago, Illinois, USA).

\section{Results}

\subsection{Characteristics of the patients}

Among the 62 patients included in the study (age $65 \pm 13$ years, $68 \%$ men), 32 (52\%) had severe AS. The valve was tricuspid in $84 \%$ of the patients and bicuspid in 10 patients. Three patients had mild renal function impairment. Patient's clinical and demographic characteristics are summarized in Table 1, and the main echocardiographic and cardiopulmonary test characteristics of the study population are presented in Tables 2 and 3, respectively.

\subsection{Functional capacity in AS}

Absolute mean peak $\mathrm{VO}_{2}$ was $19.5 \pm 5.7 \mathrm{~mL} / \mathrm{kg} / \mathrm{min}$ (median $19.6 \mathrm{~mL} / \mathrm{kg} / \mathrm{min}$; range $7.2-33.1 \mathrm{~mL} / \mathrm{kg} / \mathrm{min}$, Fig. 1). Twenty-five \% of the study population had peak $\mathrm{VO}_{2}$ values lower than $15.8 \mathrm{~mL} / \mathrm{kg} / \mathrm{min}$ and $75 \%$ lower than $22.6 \mathrm{~mL} / \mathrm{kg} / \mathrm{min}$. Forty seven \% of the study population had peak $\mathrm{VO}_{2}$ values lower than $84 \%$ of their predicted values for age and sex. Patients with reduced MEC (peak $\mathrm{VO}_{2}<$ median) were significantly older and had higher SBP values (Table 1), smaller LV end-diastolic and end-systolic volume, lower LV stroke volume, mean flow rate, cardiac output and cardiac index, lower s' and $\mathrm{e}^{\prime}$ wave velocities and higher $\mathrm{E} / \mathrm{e}^{\prime}$ ratio (Table 2). There was also a trend for a significant relationship between reduced MEC and female gender $(p=0.06)$. There were no other significant differences between the two groups regarding clinical, demographic and echocardiographic data (Tables 1 and 2). There was a significant difference between the two groups regarding peak $\mathrm{HR}, \mathrm{O}_{2}$ pulse, $\mathrm{PCP}$ and METs (Table 3 ).

There was a significant correlation between absolute peak $\mathrm{VO}_{2}$ and age $(\mathrm{r}=-0.582, \mathrm{p}<0.001)$, and body mass index $(\mathrm{r}=-0.295$, $\mathrm{p}=0.02$ ). Peak $\mathrm{VO}_{2}$ was also significantly correlated with LV stroke volume, mean flow rate, cardiac output and the indexed values of these parameters, with $\mathrm{s}^{\prime}$ (Fig. 2, Panel B) and $\mathrm{e}^{\prime}$ wave velocities, E/ $\mathrm{e}^{\prime}$ ratio (Fig. 2, Panel C) and left atrial diameter (Table 4). There was a 
Table 1

Clinical and demographic data.

\begin{tabular}{|c|c|c|c|c|}
\hline Variables & Overall $(n=62)$ & Preserved MEC $(\mathrm{n}=31)$ & Reduced MEC ( $\mathrm{n}=31)$ & $\mathrm{p}$ \\
\hline \multicolumn{5}{|l|}{ Clinical and demographic data } \\
\hline Age, years & $65 \pm 13$ & $60 \pm 13$ & $71 \pm 9$ & $<0.001$ \\
\hline Women, n (\%) & $20(32.3)$ & $6(19.4)$ & $14(45.2)$ & 0.06 \\
\hline Body surface area, $\mathrm{m}^{2}$ & $1.82 \pm 0.21$ & $1.85 \pm 0.17$ & $1.80 \pm 0.26$ & 0.38 \\
\hline Body mass index, $\mathrm{kg} / \mathrm{m}^{2}$ & $26.7 \pm 3.9$ & $25.9 \pm 3.1$ & $27.5 \pm 4.5$ & 0.11 \\
\hline Resting heart rate, bpm & $70 \pm 11$ & $73 \pm 10$ & $67 \pm 13$ & 0.08 \\
\hline Systolic blood pressure, mm Hg & $138 \pm 19.7$ & $133 \pm 16$ & $144 \pm 22$ & 0.02 \\
\hline Diastolic blood pressure, $\mathrm{mm} \mathrm{Hg}$ & $78 \pm 11$ & $76 \pm 9$ & $79 \pm 11$ & 0.25 \\
\hline \multicolumn{5}{|l|}{ Risk factors } \\
\hline Diabetes mellitus, n (\%) & $9(14.5)$ & $3(9.4)$ & $6(20.0)$ & 0.20 \\
\hline Smoking, $\mathrm{n}(\%)$ & $15(28.3)$ & $5(15.6)$ & $10(38.5)$ & 0.15 \\
\hline Dyslipidemia, n (\%) & $27(43.5)$ & $12(37.5)$ & $15(50.0)$ & 0.22 \\
\hline Hypertension, n (\%) & $27(42.2)$ & $14(43.8)$ & $13(43.3)$ & 0.84 \\
\hline Chronic renal failure, n (\%) & $3(4.8)$ & $1(3.1)$ & $2(6.7)$ & 0.48 \\
\hline
\end{tabular}

Data are presented as mean \pm SD or $n(\%)$. MEC indicates maximal exercise capacity.

trend for correlation between peak $\mathrm{VO}_{2}$ and $\mathrm{LV}$ ejection time $(\mathrm{r}=-0.23$, $\mathrm{p}=0.068$ ). There was no significant correlation between peak $\mathrm{VO}_{2}$ and global LV ejection fraction or any other parameters of stenosis severity (Table 4).

\subsection{Relationship between valvulo-arterial impedance and functional capacity}

Zva varied from $2.61 \mathrm{~mm} \mathrm{Hg} / \mathrm{mL} / \mathrm{m}^{2}$ to $7.95 \mathrm{~mm} \mathrm{Hg} / \mathrm{mL} / \mathrm{m}^{2}$ (mean = $3.97 \mathrm{~mm} \mathrm{Hg} / \mathrm{mL} / \mathrm{m}^{2}$, median $=3.82 \mathrm{~mm} \mathrm{Hg} / \mathrm{mL} / \mathrm{m}^{2}$ ). Twenty five \% of our study population had Zva values lower than $3.36 \mathrm{~mm} \mathrm{Hg} / \mathrm{mL} / \mathrm{m}^{2}$ and $25 \%$ higher than $4.3 \mathrm{~mm} \mathrm{Hg} / \mathrm{mL} / \mathrm{m}^{2}$.

Patients with reduced MEC had higher Zva as compared with those with preserved MEC (Table 2). When dividing the study population in two groups according to the median value for the Zva, $(3.82 \mathrm{~mm}$ $\mathrm{Hg} / \mathrm{mL} / \mathrm{m}^{2}$ ), patients with high Zva had higher LV end-systolic diameter
( $30 \pm 4$ vs. $27 \pm 4 \mathrm{~mL}, \mathrm{p}=0.007$ ), lower LV stroke volume ( $75 \pm 13$ vs. $85 \pm 13 \mathrm{~mL}, \mathrm{p}=0.005)$ and indexed stroke volume ( $41 \pm 5 \mathrm{vs} .47 \pm$ $7 \mathrm{~mL}, \mathrm{p}<0.001)$, lower mean flow rate $(241 \pm 44$ vs. $264 \pm 41 \mathrm{~mL} / \mathrm{s}$, $\mathrm{p}=0.037)$ and indexed flow rate $\left(131 \pm 17\right.$ vs. $147 \pm 20 \mathrm{~mL} / \mathrm{s} / \mathrm{m}^{2}$, $\mathrm{p}=0.001)$ and lower $\mathrm{e}^{\prime}$-wave velocity $(7.4 \pm 2.8$ vs. $9.0 \pm 3.1 \mathrm{~cm} / \mathrm{s}$, $\mathrm{p}=0.05$ ). Patients with high Zva were also older ( $68 \pm 11$ vs. $60 \pm$ 14 years, $\mathrm{p}=0.02$ ), had higher blood pressure (systolic $149 \pm 18 \mathrm{vs}$. $127 \pm 14 \mathrm{~mm} \mathrm{Hg}, \mathrm{p}<0.001$; diastolic $82 \pm 10$ vs. $74 \pm 9 \mathrm{~mm} \mathrm{Hg}, \mathrm{p}=$ 0.004 ) and transvalvular pressure gradient (peak $60 \pm 22$ vs. $46 \pm$ $14 \mathrm{~mm} \mathrm{Hg}, \mathrm{p}=0.006$; mean $36 \pm 15$ vs. $27 \pm 10 \mathrm{~mm} \mathrm{Hg}, \mathrm{p}=0.005)$, and smaller aortic valve area $\left(0.88 \pm 0.24\right.$ vs. $1.1 \pm 0.23 \mathrm{~cm}^{2}, \mathrm{p}=$ 0.001 ). There was a significant correlation between peak $\mathrm{VO}_{2}$ and $\mathrm{Zva}$ $(\mathrm{r}=-0.39, \mathrm{p}=0.002$; Fig. 2, Panel A) and a graded relationship between increased Zva and decreased MEC (Fig. 3). In multivariate analysis, age $(\mathrm{p}<0.001)$ and $\mathrm{Zva}(\mathrm{p}=0.048)$ were the only independent predictors $\left(\mathrm{r}^{2}=0.40\right)$ of peak $\mathrm{VO}_{2}$ (Table 5$)$.

Table 2

Echocardiographic data.

\begin{tabular}{|c|c|c|c|c|}
\hline Variables & Overall $(n=62)$ & Preserved MEC $(n=31)$ & Reduced MEC $(\mathrm{n}=31)$ & $\mathrm{p}$ \\
\hline \multicolumn{5}{|l|}{ LV geometry } \\
\hline LV end-diastolic diameter, mm & $45 \pm 6$ & $46 \pm 8$ & $45 \pm 4$ & 0.51 \\
\hline LV end-systolic diameter, mm & $28 \pm 4$ & $29 \pm 5$ & $28 \pm 4$ & 0.47 \\
\hline LV end-diastolic volume, mL & $94 \pm 22$ & $100 \pm 21$ & $89 \pm 23$ & 0.05 \\
\hline LV end-systolic volume, mL & $34 \pm 10$ & $37 \pm 12$ & $32 \pm 8$ & 0.04 \\
\hline Indexed LV mass, $\mathrm{g} / \mathrm{m}^{2}$ & $119 \pm 41$ & $118 \pm 39$ & $122 \pm 44$ & 0.71 \\
\hline \multicolumn{5}{|l|}{ LV systolic function } \\
\hline LV stroke volume, $\mathrm{mL}$ & $79 \pm 14$ & $83 \pm 14$ & $76 \pm 13$ & 0.03 \\
\hline Indexed LV stroke volume, $\mathrm{mL} / \mathrm{m}^{2}$ & $44 \pm 6$ & $45 \pm 7$ & $42 \pm 6$ & 0.07 \\
\hline Mean flow rate, $\mathrm{mL} / \mathrm{s}$ & $251 \pm 44$ & $269 \pm 43$ & $234 \pm 39$ & 0.001 \\
\hline Indexed mean flow rate, $\mathrm{mL} / \mathrm{s} / \mathrm{m}^{2}$ & $138 \pm 20$ & $146 \pm 19$ & $131 \pm 19$ & 0.003 \\
\hline Cardiac output, L/min & $5.1 \pm 0.9$ & $5.4 \pm 0.9$ & $4.8 \pm 0.8$ & 0.005 \\
\hline Cardiac index, $\mathrm{L} / \mathrm{min} / \mathrm{m}^{2}$ & $2.8 \pm 0.5$ & $2.9 \pm 0.5$ & $2.7 \pm 0.4$ & 0.018 \\
\hline LV ejection fraction, \% & $65 \pm 6$ & $64 \pm 6$ & $66 \pm 6$ & 0.42 \\
\hline $\mathrm{s}^{\prime}$-wave velocity, $\mathrm{cm} / \mathrm{s}$ & $7.8 \pm 2.0$ & $8.6 \pm 2.0$ & $7.0 \pm 0.3$ & 0.002 \\
\hline \multicolumn{5}{|l|}{ LV diastolic function } \\
\hline E wave velocity, $\mathrm{cm} / \mathrm{s}$ & $85 \pm 21$ & $82 \pm 24$ & $88 \pm 17$ & 0.29 \\
\hline E/A ratio & $1.01 \pm 0.43$ & $0.95 \pm 0.39$ & $1.03 \pm 0.42$ & 0.44 \\
\hline $\mathrm{e}^{\prime}$-wave velocity, $\mathrm{cm} / \mathrm{s}$ & $8.2 \pm 3.0$ & $9.1 \pm 3.0$ & $7.2 \pm 2.7$ & 0.011 \\
\hline $\mathrm{E} / \mathrm{e}^{\prime}$ ratio & $11.7 \pm 5.1$ & $9.8 \pm 4.2$ & $13.8 \pm 5.4$ & 0.003 \\
\hline \multicolumn{5}{|l|}{ Left atrial geometry } \\
\hline Left atrial diameter, mm & $37 \pm 5$ & $36 \pm 5$ & $38 \pm 5$ & 0.15 \\
\hline \multicolumn{5}{|l|}{ Assessment of LV load } \\
\hline Peak trans-aortic pressure gradient, mm Hg & $53 \pm 20$ & $54 \pm 17$ & $52 \pm 23$ & 0.62 \\
\hline Mean trans-aortic pressure gradient, $\mathrm{mm} \mathrm{Hg}$ & $31 \pm 13$ & $33 \pm 12$ & $30 \pm 15$ & 0.45 \\
\hline Aortic valve area, $\mathrm{cm}^{2}$ & $0.99 \pm 0.26$ & $1.03 \pm 0.25$ & $0.95 \pm 0.27$ & 0.28 \\
\hline Indexed aortic valve area, $\mathrm{cm}^{2} / \mathrm{m}^{2}$ & $0.54 \pm 0.13$ & $0.55 \pm 0.13$ & $0.53 \pm 0.14$ & 0.41 \\
\hline Valvulo-arterial impedance, $\mathrm{mm} \mathrm{Hg} / \mathrm{ml} / \mathrm{m}^{2}$ & $3.97 \pm 0.97$ & $3.71 \pm 0.68$ & $4.24 \pm 1.18$ & 0.036 \\
\hline
\end{tabular}

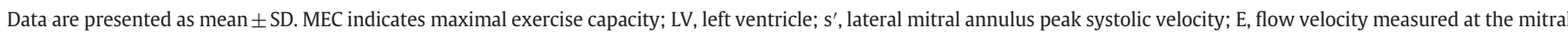
leaflets tip; e', lateral mitral annulus peak early diastolic velocity. 
Table 3

Cardiopulmonary exercise test data.

\begin{tabular}{|c|c|c|c|c|}
\hline Variables & Overall $(n=62)$ & Preserved MEC $(n=31)$ & Reduced MEC $(\mathrm{n}=31)$ & $\mathrm{p}$ \\
\hline Peak heart rate, bpm & $131 \pm 21$ & $140 \pm 19$ & $119 \pm 17$ & $<0.001$ \\
\hline Peak SBP, mm Hg & $177 \pm 24$ & $180 \pm 21$ & $175 \pm 27$ & 0.43 \\
\hline Peak DBP, mm Hg & $83 \pm 12$ & $85 \pm 11$ & $81 \pm 11$ & 0.13 \\
\hline $\mathrm{PCP}, \mathrm{mm} \mathrm{Hg}{ }^{*} \mathrm{~mL} / \mathrm{kg} / \mathrm{min}$ & $3456 \pm 1154$ & $4317 \pm 820$ & $2652 \pm 779$ & $<0.001$ \\
\hline METs & $5.7 \pm 1.7$ & $6.8 \pm 1.3$ & $4.7 \pm 1.2$ & $<0.001$ \\
\hline $\mathrm{O}_{2}$ pulse, $\mathrm{mL} /$ beat & $11.1 \pm 3.3$ & $11.8 \pm 3.1$ & $10.1 \pm 3.2$ & 0.05 \\
\hline
\end{tabular}

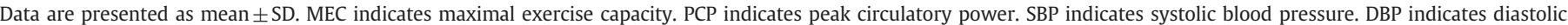
blood pressure. METs indicates metabolic equivalents. $\mathrm{O}_{2}$ pulse indicates oxygen pulse.

\section{Discussion}

The major findings of the present study are: 1) MEC varies widely in asymptomatic patients with moderate to severe AS and is often lower than expected; about half of the study population had peak $\mathrm{VO}_{2}$ values lower than those expected for their age and body weight, 2) MEC is not influenced by classical parameters of AS severity, 3) MEC is modestly related to the degree of LV systolic and diastolic dysfunction and 4) reduced MEC is independently associated with older age and higher global LV hemodynamic load.

\subsection{Functional capacity in AS}

Our results confirm and reinforce the findings of other smaller studies showing that MEC is frequently impaired in patients with AS $[7,8]$. Clyne et al. studied LV response to exercise by assessing peak oxygen uptake in 14 asymptomatic patients with AS, and found that these patients had reduced exercise tolerance as compared with controls [7]. Recently, Rajani et al. have showed, among 38 patients with AS, that those who developed symptoms during CPET had lower peak $\mathrm{VO}_{2}$ and reduced peak indexed stroke volumes [8]. In the whole cohort MEC varied widely from normal to markedly reduced values. The mean peak $\mathrm{VO}_{2}(19.5 \pm 5.7 \mathrm{~mL} / \mathrm{kg} / \mathrm{min})$ in the present study was however lower than in the study of Clyne et al. $(26.7 \pm 6.3 \mathrm{~mL} / \mathrm{kg} / \mathrm{min})$. This is partly explained by the difference in age between the two cohorts. Of note, age was an important determinant of peak $\mathrm{VO}_{2}$ in our study, while female sex was only weakly associated with MEC. In this regard, a recent study emphasized the close relationship between age and reduced exercise capacity, as assessed by exercise duration in AS patients [9]. Furthermore, patients with diminished MEC had higher systolic blood pressure at rest. In elderly subjects, it is well known that systolic blood pressure is often increased, partly as a result of changes in systemic

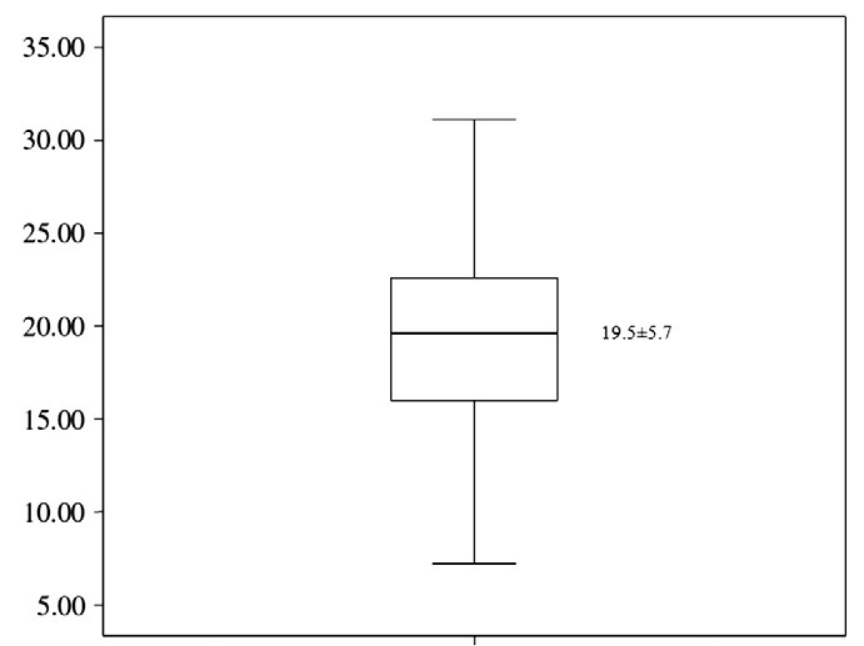

Peak $\mathrm{VO}_{2}, \mathrm{~mL} / \mathrm{kg} / \mathrm{min}$

Fig. 1. Maximal exercise capacity in patients with aortic stenosis. arterial mechanical properties with increased arterial stiffness [10,11]. The combination of systolic hypertension and of aortic valve stenosis may yield to a markedly increased LV global hemodynamic burden, which may in turn negatively impact myocardial oxygen balance and MEC.

\subsection{Echocardiographic predictors of functional capacity in AS}

In the Fick equation, peak $\mathrm{VO}_{2}$ is related to cardiac output. On exercise, a similar relationship exists. In our study, the correlation found between peak $\mathrm{VO}_{2}$ and stroke volume at rest and the derived cardiac output is consistent with the Fick equation. The higher the cardiac output at rest, the easier the LV adaption to exercise, and the higher the maximum oxygen uptake. We found a similar linear relationship with resting mean transvalvular flow rate, suggesting that reduced MEC is, at least in part, determined by the impairment of LV pump function, which is, in turn, dependent on the extent of myocardial systolic dysfunction and degree of LV concentric remodelling. To this effect, in this study there was a good correlation between longitudinal LV systolic function - as assessed by tissue Doppler velocities - and peak $\mathrm{VO}_{2}$. Longitudinal function is governed by the sub-endocardial myocardial fibers [12], which are more likely to be affected by excessive intracavitary pressure and microvascular ischemia [13]. In AS, longitudinal systolic function can be reduced despite preserved LV ejection fraction $[14,15]$. Indeed, LV ejection fraction markedly underestimates the extent of myocardial systolic dysfunction in presence of LV concentric remodelling and is therefore often normal in AS patients. In asymptomatic AS, impaired sub-endocardial function as reflected by reduced LV longitudinal function has been shown to be associated with impaired exercise tolerance [16] and development of symptoms in the short-term $[17,18]$. Our results extend these previous findings. As expected, LV ejection fraction did not influence the exercise capacity in this study. Reduced exercise capacity was also univariately associated with 1 ) enlarged left atrium - a marker of chronic diastolic burden, 2) impaired LV relaxation - as assessed by early diastolic annular velocity $\left(\mathrm{e}^{\prime}\right)$, and 3 ) increased $\mathrm{E} / \mathrm{e}^{\prime}$ - an estimate of LV filling pressure. All these parameters reflect the extent of LV diastolic dysfunction. Our data are in line with previous findings reported in various cardiovascular diseases [19-23].

\subsection{LV global hemodynamic load and functional capacity in AS}

Patients with calcific AS often have a double hemodynamic load: a valvular load resulting from the AS and an arterial load that is frequently increased in these patients due to the high prevalence of atherosclerosis and ageing-related medial elastoclacinosis [3]. These findings outline the complex interplay between the ventricular, vascular and valvular components in AS. The increase in the global LV hemodynamic load may affect the LV adaptation to exercise and thereby limit the MEC. In our study, Zva - an estimate of global LV load - was significantly correlated with peak $\mathrm{VO}_{2}$. When Zva increases, the LV has to generate more pressure per milliliter of blood ejected during systole, thus reducing its maximum capacity to maintain adequate flow to satisfy tissue metabolic requirements during exercise. Of note, none of the classical parameters of AS severity 

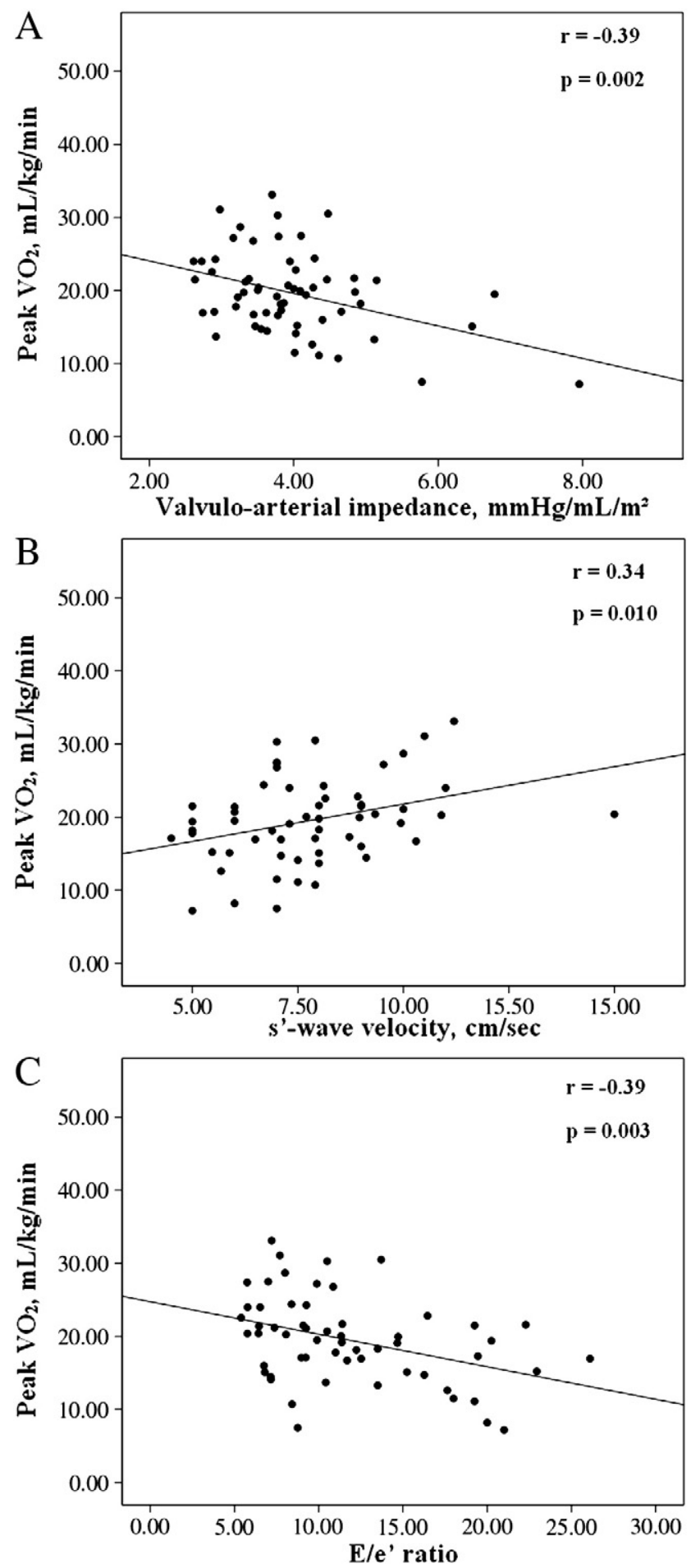

Fig. 2. Relationship between maximal exercise capacity $\left(\right.$ Peak $\mathrm{VO}_{2}$ ) and global left ventricular (LV) hemodynamic load (Panel A), LV longitudinal function as assessed by s'-wave velocity (Panel B) and estimated LV filling pressure (Panel C).

(valve area, pressure gradients, aortic jet velocity) were significantly related to MEC. This suggests that the global LV hemodynamic load estimated with the use of Zva better reflects the consequences of the valvulo-arterial disease on the myocardial function and exercise capacity. Recent studies reported that Zva enables to identify asymptomatic AS patients at risk of rapid progression to symptoms and worse outcomes. A valvulo-arterial impedance $>5 \mathrm{~mm} \mathrm{Hg} / \mathrm{ml} / \mathrm{m}^{2}$ has been found to be independently associated with a 4 -fold increase in risk of LV systolic dysfunction and a value of $>4.5 \mathrm{~mm} \mathrm{Hg} / \mathrm{ml} / \mathrm{m}^{2}$ was
Table 4

Relationship between peak $\mathrm{VO}_{2}$, age and echocardiographic parameters.

\begin{tabular}{lrr}
\hline Variables & $\mathrm{R}$ & $\mathrm{p}$ \\
\hline Age & -0.58 & $<0.001$ \\
LV geometry & & \\
LV end-diastolic diameter & 0.03 & 0.795 \\
LV end-systolic diameter & 0.08 & 0.525 \\
LV end-diastolic volume & 0.18 & 0.171 \\
LV end-systolic volume & 0.14 & 0.270 \\
Indexed LV mass & -0.05 & 0.652 \\
LV systolic function & & \\
LV stroke volume & 0.27 & 0.035 \\
Indexed LV stroke volume & 0.31 & 0.014 \\
Mean flow rate & 0.37 & 0.003 \\
Indexed mean flow rate & 0.44 & $<0.001$ \\
Cardiac output & 0.32 & 0.011 \\
Cardiac index & 0.35 & 0.006 \\
LV ejection fraction & -0.06 & 0.601 \\
S'-wave velocity & 0.34 & 0.010 \\
LV diastolic function & & \\
E-wave velocity & -0.11 & 0.388 \\
E/A ratio & -0.08 & 0.520 \\
e'-wave velocity & 0.32 & 0.013 \\
E/e' ratio & -0.39 & 0.003 \\
Left atrial geometry & & \\
Left atrial diameter & -0.26 & 0.048 \\
Assessment of LV load & & \\
Peak transaortic pressure gradient & -0.01 & 0.948 \\
Mean transaortic pressure gradient & 0.02 & 0.855 \\
Aortic valve area & 0.12 & 0.337 \\
Indexed aortic valve area & 0.14 & 0.264 \\
Valvulo-arterial impedance & -0.39 & 0.002 \\
\hline
\end{tabular}

LV indicates left ventricle; $s^{\prime}$, lateral mitral annulus peak systolic velocity; E, flow velocity measured at the mitral leaflets tip; $\mathrm{e}^{\prime}$, lateral mitral annulus peak early diastolic velocity.

associated with a 2.5 -fold increase in risk of mortality in patients with AS [24]. In our study, the negative impact of Zva on peak $\mathrm{VO}_{2}$ became significant for values $>4 \mathrm{~mm} \mathrm{Hg} / \mathrm{ml} / \mathrm{m}^{2}$, and the magnitude of this impact increased with increasing Zva. Further studies are needed to assess whether limited exercise capacity, as evaluated during CPET, is of prognostic significance in asymptomatic AS.

\section{Limitations}

Peak $\mathrm{VO}_{2}$ value normalized for body weight in kilograms was used and this may underestimates peak $\mathrm{VO}_{2}$ in obese subjects. However, in our patients, mean body mass index was $26.7 \pm 3.9$, and only $7(11 \%)$ were obese. Peak $\mathrm{VO}_{2}$ may also be affected by other clinical conditions such as pulmonary diseases, neuromuscular or musculoskeletal disease, anemia or any other pathology that decreases the $\mathrm{O}_{2}$ transport capacity of blood. However, none of the patients included in the

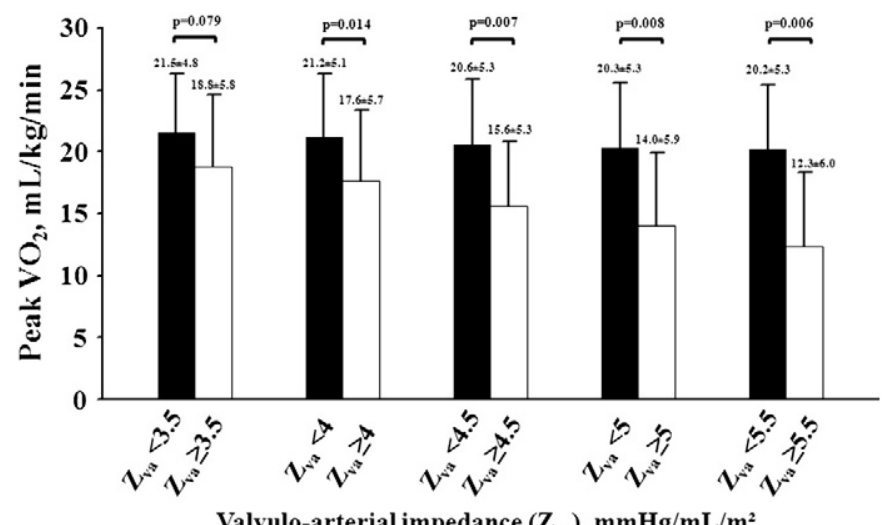

Fig. 3. Variation of maximal exercise capacity according to various cut-off values of global left ventricular hemodynamic load. 
Table 5

Multiple linear regression analysis for prediction of maximal exercise capacity (total $\left.r^{2}=0.40\right)$.

\begin{tabular}{lrlr}
\hline & \multicolumn{1}{l}{ B } & \multicolumn{1}{l}{ SE } & \multicolumn{1}{l}{$\mathrm{P}$} \\
\hline $\mathrm{s}^{\prime}$-wave velocity & 0.039 & 0.428 & 0.927 \\
E/e' ratio & -0.164 & 0.133 & 0.224 \\
LA diameter & -0.043 & 0.166 & 0.796 \\
Age & -0.235 & 0.055 & $<0.001$ \\
Valvulo-arterial impedance & -1.383 & 0.680 & 0.048 \\
\hline
\end{tabular}

$\mathrm{S}^{\prime}$ indicates lateral mitral annulus peak systolic velocity; LA, left atrium; $\mathrm{E}$, indicates flow velocity measured at mitral leaflets tip; $e^{\prime}$, lateral mitral annulus peak early diastolic velocity.

present study had symptoms or a medical history suggesting any of the mentioned pathologies.

The MEC was evaluated using treadmill exercise testing, not cycle ergometer. In this regard, our results cannot be extrapolated to all modalities used to measure MEC.

Peak $\mathrm{VO}_{2}$ values measured using treadmill exercise testing are generally higher than those measured with cycle ergometer. We have reported a $47 \%$ of "abnormally reduced" MEC, as assessed by the age- and weight-predicted peak $\mathrm{VO}_{2}$. Consequently, we may expect that this percent would be even higher by using cycle ergometer. Exercise-induced myocardial ischemia may be a limiting factor of exercise capacity in these patients. However, none of our patients had a history of coronary artery disease, or presented during exercise angina or significant ST changes suggestive of active myocardial ischemia.

\section{Conclusion}

In asymptomatic patients with moderate to severe asymptomatic AS, functional capacity varies widely among patients, and is often lower than expected. MEC was not related to the classical indices of stenosis severity (peak velocity, gradient, AVA). MEC was related to age, parameters of LV hemodynamic load, diastolic function, and systolic pump function. Global LV hemodynamic load as estimated by the Zva was the main independent echocardiographic determinant of reduced MEC in these patients, suggesting the usefulness of this parameter for risk stratification and management of these patients.

\section{Acknowledgments}

We thank Carmine Celentano for the excellent technical assistance.

\section{References}

[1] Pibarot P, Dumesnil JG. Assessment of aortic stenosis severity: check the valve but don't forget the arteries. Heart 2007;93(7):780-2.

[2] Rajamannan NM, Bonow RO, Rahimtoola SH. Calcific aortic stenosis: an update. Nat Clin Pract Cardiovasc Med 2007:4(5):254-62.
[3] Briand M, Dumesnil J, Kadem L, et al. Reduced systemic arterial compliance impacts significantly on left ventricular afterload and function in aortic stenosis: implications for diagnosis and treatment. J Am Coll Cardiol 2005;46(2):291-8.

[4] Das P, Rimington H, Chambers J. Exercise testing to stratify risk in aortic stenosis. Eur Heart J 2005;26(13):1309-13.

[5] Alborino D, Hoffmann JL, Fournet PC, Bloch A. Value of exercise testing to evaluate the indication for surgery in asymptomatic patients with valvular aortic stenosis. J Heart Valve Dis 2002;11(2):204-9.

[6] ATS/ACCP Statement on Cardiopulmonary Exercise Testing. Am J Respir Crit Care Med 2003;167(2):211-77.

[7] Clyne C, Arrighi J, Maron B, Dilsizian V, Bonow RO, Cannon III RO. Systemic and left ventricular responses to exercise stress in asymptomatic patients with valvular aortic stenosis. Am J Cardiol 1991;68(15):1469-76.

[8] Rajani R, Rimington H, Chambers J. Treadmill exercise in apparently asymptomatic patients with moderate or severe aortic stenosis:relationship between cardiac index and revealed symptoms. Heart 2010;96(9):689-95.

[9] Rajani R, Rimington H, Nabeebaccus A, Chowienczyk P, Chambers JB. Asymptomatic aortic stenosis: the influence of the systemic vasculature on exercise time. J Am Soc Echocardiogr 2012;25(6):613-9.

[10] Lakatta EG, Levy D. Arterial and cardiac aging: major shareholders in cardiovascular disease enterprises: Part I: aging arteries: a "set up" for vascular disease. Circulation 2003;107(1):139-46.

[11] Lakatta EG, Levy D. Arterial and cardiac aging: major shareholders in cardiovascular disease enterprises: Part II: the aging heart in health: links to heart disease. Circulation 2003;107(2):346-54.

[12] Greenbaum RA, Ho SY, Gibson DG, Becker AE, Anderson RH. Left ventricular fibre architecture in man. Br Heart J 1981;45(3):248-63.

[13] Ohtsuka S, Kakihana M, Watanabe H, Enomoto T, Ajisaka R, Sugishita Y. Alterations in left ventricular wall stress and coronary circulation in patients with isolated systolic hypertension. J Hypertens 1996;14(11):1349-55.

[14] Ng A, Delgado V, Bertini M, et al. Alterations in multidirectional myocardial functions in patients with aortic stenosis and preserved ejection fraction: a two-dimensional speckle tracking analysis. Eur Heart J 2011;32(12):1542-50.

[15] Tongue AG, Dumesnil JG, Laforest I, Theriault C, Durand LG, Pibarot P. Left ventricular longitudinal shortening in patients with aortic stenosis: relationship with symptomatic status. J Heart Valve Dis 2003:12:142-9.

[16] Lafitte S, Perlant M, Reant P, et al. Impact of impaired myocardial deformations on exercise tolerance and prognosis in patients with asymptomatic aortic stenosis. Eur J Echocardiogr 2009 May;10(3):414-9.

[17] Lancellotti P, Donal E, Magne J, et al. Risk stratification in asymptomatic moderate to severe aortic stenosis: the importance of the valvular, arterial and ventricular interplay. Heart 2010;96(17):1364-71.

[18] Lancellotti P, Moonen M, Magne J, et al. Prognostic effect of long-axis left ventricular dysfunction and B-type natriuretic peptide levels in asymptomatic aortic stenosis. Am J Cardiol 2010;105(3):383-8.

[19] Dalsgaard M, Kjaergaard J, Pecini R, et al. Predictors of exercise capacity and symptoms in severe aortic stenosis. Eur J Echocardiogr 2010;11(6):482-7.

[20] Skaluba S, Litwin S. Mechanisms of exercise intolerance: insights from tissue Doppler imaging. Circulation 2004;109(8):972-7.

[21] Matsumura Y, Elliot P, Virdee M, Sorajja P, Doi Y, McKenna WJ. Left ventricular diastolic function assessed using Doppler tissue imaging in patients with hypertrophic cardiomyopathy: relation to symptoms and exercise capacity. Heart 2002;87(3): 247-51.

[22] Hadano Y, Murata K, Yamamoto T, et al. Usefulness of mitral annular velocity in predicting exercise tolerance in patients with impaired left ventricular systolic function. Am J Cardiol 2006;97(7):1025-8.

[23] Smart N, Haluska B, Leano R, Case C, Mottram PM, Marwick TH. Determinants of functional capacity in patients with chronic heart failure: role of filling pressure and systolic and diastolic function. Am Heart J 2005;149(1):152-8.

[24] Hachicha Z, Dumesnil JG, Pibarot P. Usefulness of the valvuloarterial impedance to predict adverse outcome in asymptomatic aortic stenosis. J Am Coll Cardiol 2009;54(11):1003-11. 